\title{
Exponential Divergence and Long Time Relaxation in Chaotic Quantum Dynamics
}

\author{
Arjendu K. Pattanayak and Paul Brumer \\ Chemical Physics Theory Group, University of Toronto, Toronto, Canada M5S 3 H6
}

(Received 5 January 1996)

\begin{abstract}
Phase-space representations of the dynamics of the quantal and classical cat map are used to explore quantum-classical correspondence in a $K$ system: As $\hbar \rightarrow 0$, the classical chaotic behavior is shown to emerge smoothly and exactly. The quantum dynamics near the classical limit displays both exponential separation of adjacent distributions and long time relaxation, two characteristic features of classical chaotic motion. [S0031-9007(96)00558-3]
\end{abstract}

PACS numbers: 05.45.+b, 03.65.Sq, 05.40.+j

Understanding the correspondence principle for bound conservative systems which are classically chaotic presents a serious challenge [1]. The classical system is characterized by positive $K$ entropy which is reflected, for example, in the exponential divergence of initially nearby trajectories and in the relaxation [2] of distributions in the long-term limit. By contrast, the quantum dynamics is not even ergodic, and displays long time recurrences. Current views on correspondence in conservative chaotic systems range from simple belief in the correspondence principle, to reliance on decoherence induced by coupling to an environment [3], to calls for the overthrow of quantum mechanics due to its purported inability to display chaos in the correspondence limit [1]. Resolving this controversy requires studies of quantum dynamics close to the classical limit, a task thus far made impossible by numerical difficulties as $\hbar \rightarrow 0$.

In this Letter we demonstrate, using numerical results based on an exact quantum propagator [4], the approach to the classical limit of the chaotic Arnold cat [5]. The nonsingular character of our phase space propagator as $\hbar \rightarrow 0$ allows direct insight into correspondence in this bounded chaotic system. In particular, we show that quantum mechanics does display chaos as $\hbar \rightarrow$ 0 , including exponential divergence of initially nearby distributions and long time relaxation. The agreement between quantum and classical dynamics extends over longer times as $\hbar$ decreases [6].

Classical-quantum correspondence is best examined [7] in the Liouville picture classically and in some phase space representation of the density matrix (e.g., the Wigner representation [8]) quantum mechanically. This approach provides an overlap of objects and concepts which does not exist if one attempts to utilize, for example, classical trajectories and quantum wave functions. The advantages of this approach are evident in the results below where operationally chaotic behavior is demonstrated in quantum mechanics.

The classical cat map is a $K$ system which corresponds to the classical dynamics of a kicked oscillator with Hamiltonian [9]

$$
H=p^{2} / 2 \mu+\epsilon x^{2} / 2 \sum_{s=-\infty}^{\infty} \delta(s-t / T)
$$

restricted to a torus $0 \leq x<a, 0 \leq p<b$. The proper quantization of this system and its approach to the classical limit has been extensively considered [4,9,10]. Early quantizations [10] of this system were unsettling insofar as (a) only a restricted class of classical tori (those with $a b=h N, N$ integer) could be quantized, (b) the solutions violated the uncertainty principle, and (c) the quantum propagator thus obtained did not reduce to the classically chaotic cat map in the $\hbar \rightarrow 0$ limit.

A recently introduced [4] phase space quantization eliminated these problems and yielded an analytic quantum propagator. Specifically, the one time-step quantum propagation of the Wigner-Weyl representation of the quantal density operator $\rho^{Q}(x, p ; t)$ in phase space $(x, p)$ is given by

$$
\rho^{Q}(x, p ; T)=\frac{1}{a b} \sum_{n, m=-\infty}^{\infty} \rho_{n, m}^{Q}(T) f_{n, m},
$$

where

$$
\rho_{n, m}^{Q}(T)=\sum_{k, l=-\infty}^{\infty} G(n, m ; k, l) \rho_{k, l}^{Q}(0) .
$$

The propagator $G$ of the Fourier coefficients is given by

$$
\begin{aligned}
G(n, m ; k, l)= & e^{i \pi \alpha(k l-n m)} \int_{0}^{1} d \nu \int_{0}^{1} d \nu^{\prime} e^{-i \pi \xi[(\nu+\alpha n) \bmod 1]^{2} / \alpha} e^{i \pi \xi \nu^{2} / \alpha} \\
& \times e^{2 \pi i(l-m) \nu} e^{-i \pi \eta\left[\left(\nu^{\prime}+\alpha l\right) \bmod 1\right]^{2} / \alpha} e^{i \pi \eta \nu^{\prime 2} / \alpha} e^{2 \pi i(k-n) \nu^{\prime}},
\end{aligned}
$$

where $\eta=T b / \mu a \in \mathbf{Z}$ (the set of integers) and $\xi=$ $-\epsilon T a / b \in \mathbf{Z}$ obtain in scaling the original Hamiltonian to the unit torus, $\alpha=h / a b$ acts as a dimensionless form of Planck's constant for this problem, and $f_{n, m}(p, x)=$ $\exp \{2 \pi i(n p / a+m x / b)\}$ is the Fourier expansion basis. The Arnold cat map [5] corresponds to the choice $\eta=\xi=1$. Equation (4) can be integrated exactly [11] to provide an analytic propagator for all $\hbar$. This result 

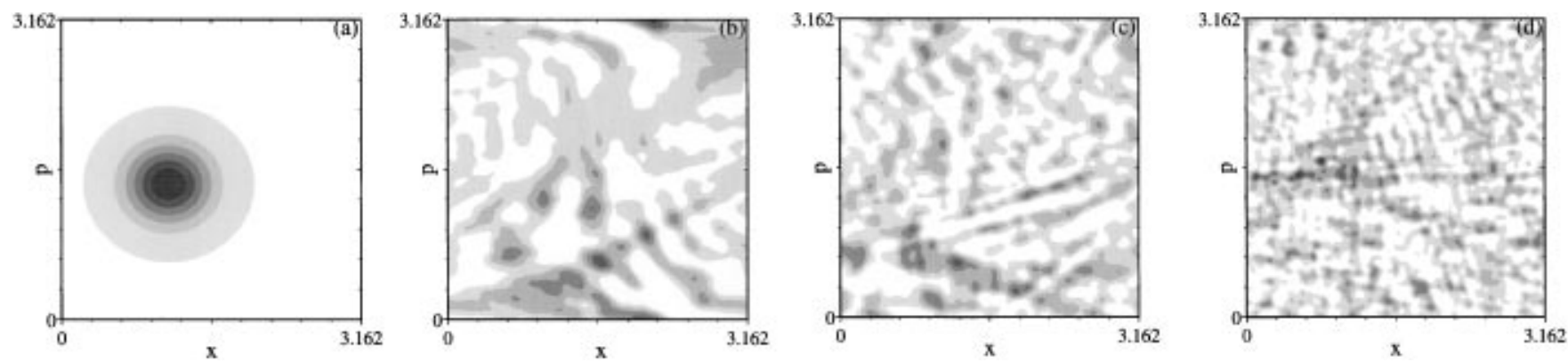

FIG. 1. Snapshots of $\left|\rho^{Q}(x, p, t)\right|$. Densities are represented by five shadings of gray denoting five evenly spaced probabilities between $7 \times 10^{-5}$ to $1 \times 10^{-6}$. (a) $t=0$, (b) $t=2 T$, (c) $t=4 T$, (d) $t=8 T ; \alpha=0.1(\lambda a=\lambda b=3.162)$.

contrasts with the classical propagator given by Eq. (2) but with $\rho_{n, m}^{Q}(T)$ replaced by the classical form

$$
\begin{gathered}
\rho_{n, m}(T)=\sum_{k, l=-\infty}^{\infty} G_{c}(n, m ; k, l) \rho_{k, l}(0), \\
G_{c}(n, m ; k, l)=\delta_{(k, l), \phi^{T} \cdot(n, m) .}
\end{gathered}
$$

Here $\phi^{T} \cdot(n, m)$ is the vector resulting from premultiplying the vector $(n, m)$ with the transpose $\phi^{T}$ of the matrix governing the point dynamics:

$$
\begin{aligned}
\left(\begin{array}{c}
\tilde{x}_{n+1} \\
\tilde{p}_{n+1}
\end{array}\right) & =\phi\left(\begin{array}{c}
\tilde{x}_{n} \\
\tilde{p}_{n}
\end{array}\right) \bmod 1 \\
& =\left(\begin{array}{cc}
1 & \eta \\
\xi & 1+\eta \xi
\end{array}\right)\left(\begin{array}{c}
\tilde{x}_{n} \\
\tilde{p}_{n}
\end{array}\right) \bmod 1,
\end{aligned}
$$

with $\tilde{x}=x / a, \tilde{p}=p / b$.

Note that, by contrast with the classical case, the quantum propagator $G$ mixes contributions from all $k, l$ components to produce each $n, m$. It shares with the classical propagator a fixed point at $(n, m)=(0,0)$. However, it also has an infinite number of additional fixed points for rational $\alpha=u / v$; these lie in the quantal Fourier space at all $(w v, z v)$ with $u, v, w, z \in \mathbf{Z}$. These fixed points affect the quantal evolution of a distribution in the following ways: (a) Near the classical limit ( $\alpha \ll 1$ or $v \gg u \geq$ $1)$, these fixed points are far out in the Fourier space, and hence affect structure at extremely small scales. Thus, it is in the highly oscillatory regime (whether the structure is prepared initially or acquired during the evolution) that the dynamics will exhibit quantal evolution distinct from the classical dynamics. (b) At larger $\alpha$, these fixed points are both more dense in the Fourier space and exist at smaller values of $(n, m)$, indicating a far more substantial deviation from the classical evolution. (c) Even though there are no such demonstrable fixed points for irrational $\alpha$, the propagator is a smooth function of $\alpha$; the qualitative conclusions from these arguments should therefore extend to all $\alpha$.

The evolution of distributions which are initially of the Gaussian form

$$
N_{\lambda} \exp \left[-\left(x-\lambda x_{0}\right)^{2} / \lambda^{2} \sigma_{x}^{2}\right] \exp \left[-\left(p-\lambda p_{0}\right)^{2} / \lambda^{2} \sigma_{p}^{2}\right]
$$

is displayed below. Here $N_{\lambda}$ is a normalization factor, $\left(\lambda x_{0}, \lambda p_{0}\right)$ specifies the location of the Gaussian of width $\left(\lambda \sigma_{x}, \lambda \sigma_{p}\right)$ in a phase space of dimension $\lambda^{2} a b$. The variable scaling factor $\lambda$ can be used to approach the classical limit. That is, as $\lambda$ increases the volume of phase space $\lambda^{2} a b$ increases and $\alpha=h / \lambda^{2} a b$, the effective Planck's constant of the problem, decreases. Hence, by increasing $\lambda$ we approach the classical limit while preserving the ratio $\left(\lambda^{2} \sigma_{x} \sigma_{p} / \lambda^{2} a b\right)$ of the volume of the initial distribution to the volume of phase space. We note that we work in units such that $h=1$.

Figures 1-3 show gray-scale contour maps of the evolution [12] of the Wigner function for $\alpha=10^{-1}, 10^{-2}$, and $10^{-5}$. To simplify the presentation we report the absolute value of the Wigner representation of the density. The Wigner-Weyl function for $\alpha=10^{-5}$ is always positive definite and numerically identical to the classical Liouville density over the indicated time scale. We note
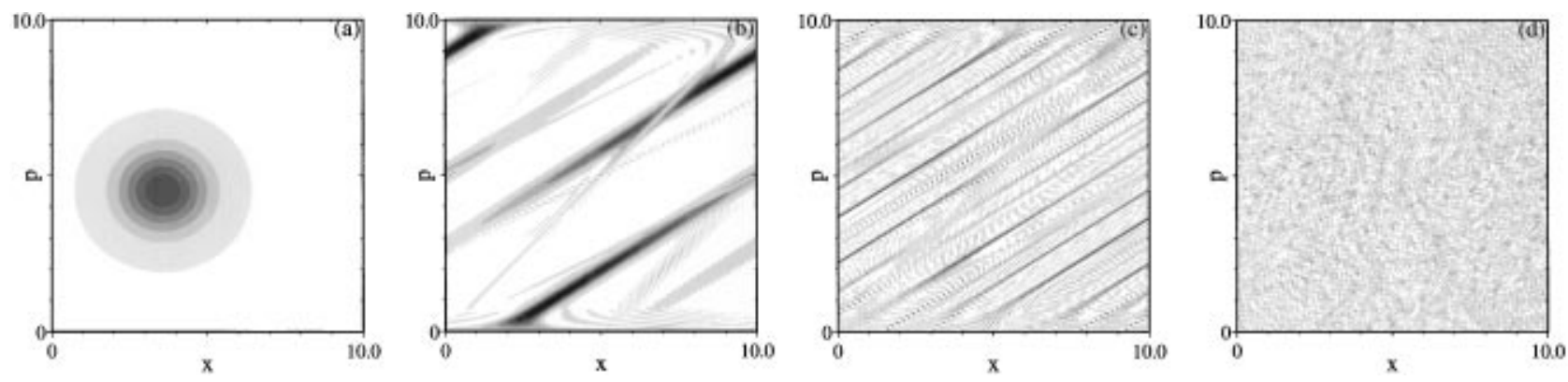

FIG. 2. As in Fig. 1 but with $\alpha=10^{-2}(\lambda a=\lambda b=10)$. 

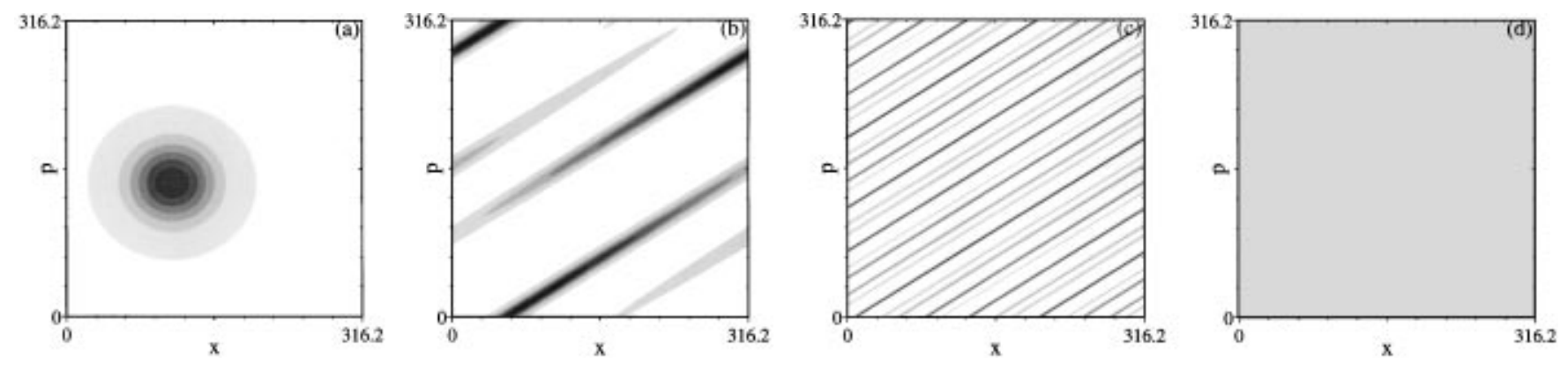

FIG. 3. As in Fig. 1 but with $\alpha=10^{-5}(\lambda a=\lambda b=316.2)$.

that the classical dynamics is scale invariant; hence the classical phase-space figures are independent of $\lambda$ and are the same for all three cases and identical to the $\alpha=10^{-5}$ case. The analytic results [4] ensure that the $\alpha \rightarrow 0$ limit is indeed classical mechanics, but these computations allow a direct examination of the approach to this limit.

For $\alpha=0.1$ (Fig. 1) the system is seen to be in the deep quantum regime. The propagating wave packet shows only the barest resemblance to the classical result, and interference structures dominate. There is no relaxation of the dynamics over the indicated time scale, and the expectation value of operators (not shown) oscillate with large amplitude. Although not evident in this figure, the Wigner distribution has many positive and negative regions. For $\alpha=0.01$ (Fig. 2) the classical density striations begin to appear, although still accompanied by quantum effects. Various parts of the distributions appear to interfere with one another, giving interference fringes. Once again, relaxation is not evident after eight time steps, although a large coarse graining will result in a uniform phase-space distribution. Separate calculations show that the expectation value of operators in this $\alpha$ regime display small fluctuations about the equilibrium value. Finally, as noted above, the $\alpha=10^{-5}$ result agrees exactly with the figures from the classical evolution: The distribution has relaxed [2] even over the short time scale shown. The approach to classical mechanics is clearly smooth and devoid of singularities.

Relaxation is but one characteristic of chaotic motion, evident at long times. Classical chaos, however, is typically manifested in the short time instability associated with exponential divergence of adjacent trajectories. Standard arguments [13] imply that such exponential separation is not possible in quantum mechanics. Here we demonstrate that this is not the case; we have obtained similar exponential divergence for compact phase space distributions, both classically and quantally close to the classical limit. In the absence of point trajectories and an appropriate tangent space, we examine the time dependence of the distance $\Delta$ between two nearby, initially well localized Gaussians [14]; the results are shown in Fig. 4. Also shown for comparison is a straight line with slope dictated by the $K$ entropy of the classical cat map; since the system is uniformly hyperbolic, this is exactly equal to the Lyapunov exponent of the underlying point dynam- ics. The distance in phase space between the two distributions is determined by the centroids: $\Delta=\left[\left(\langle x\rangle_{1}-\right.\right.$ $\left.\left.\langle x\rangle_{2}\right)^{2}+\left(\langle p\rangle_{1}-\langle p\rangle_{2}\right)^{2}\right]^{1 / 2}$, where \langle\rangle$_{i}$ denotes the average over the $i$ th distribution $(i=1,2)$. The quantity $\Delta(t)$ was evaluated both classically and quantum mechanically for various values of $a, b$.

The time dependence of $\Delta$ was found to be essentially identical in classically and quantally propagated distributions over times up to eight steps, for the $a, b$ (and hence $\alpha$ ) in the range shown. At early times, dependent on $\alpha$, $\Delta$ increases essentially exponentially (see Fig. 4) with increasing adherence to the classical $K$-entropy line as $\alpha$ is reduced. At times longer than that shown in Fig. 4, $\Delta$ tends to decrease in a generally similar way quantum mechanically and classically, a consequence of saturation [15] and relaxation in the finite size phase space. When $\alpha$ is increased, since there is no relaxation, $\Delta$ continues to oscillate at larger times quantum mechanically, whereas the classical dynamics relaxes. The combination of short time exponential divergence and long time relaxation demonstrates the loss of information characteristic of chaos for both the classical and near-classical quantal dynamics.

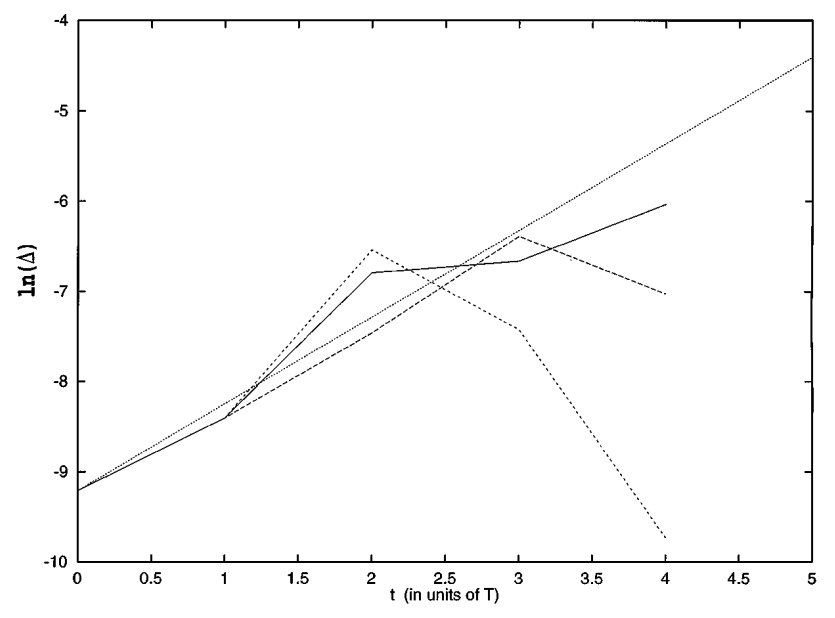

FIG. 4. Short time exponential separation of centroids for $a=b=50.0\left(\alpha=4 \times 10^{-4}\right) \quad$ (dashed), $a=b=28.79\left(\alpha=1.207 \times 10^{-3}\right) \quad$ (solid), $\quad a=b=$ $16.57\left(\alpha=3.64 \times 10^{-2}\right)$ (dotted). See text for other parameters. Similar results are obtained by propagating the same distributions classically. The straight line with a slope of 0.9624 shows classical exponential separation of adjacent point trajectories. 
Finally, we note that the availability of an analytic quantum propagator for a $K$ system allows a study of the expected time dependence of classical-quantum agreement as a function of $\hbar$. Preliminary results suggest that increasing the value of $\alpha$ does, in general, decrease the time scale over which quantum and classical dynamics agree. However, the extent of agreement also depends strongly on various other factors, including the nature of the initial distribution. The variation within the quantumclassical deviations for different initial conditions at a given value of $\alpha$ and $t$ spans many orders of magnitude. Further studies on this issue await application of our general quantization approach to classical maps which have smaller $K$ entropy than the classical cat map. That is, the large value of the $K$ entropy for the cat map forces relaxation of the dynamics over a rather short time. Further, it also rapidly saturates the exponential divergence (Fig. 4) which could otherwise extend to significantly longer times.

In summary, although the literature suggests that demonstrating quantum-classical correspondence becomes increasingly difficult (if not impossible [1,9]) with increasing classical chaos, we have been able to show this correspondence in a classical $K$ system. We have also shown how correspondence emerges smoothly from the quantal behavior as $\alpha \rightarrow 0$. These results make clear that the dynamics of distributions in the nonergodic quantal cat map display the essential attributes of the classical $K$ system as the system approaches the classical limit. They constitute the first demonstration of the emergence of characteristically classically chaotic behavior from quantum mechanically nonergodic behavior as one approaches this limit.

This work was supported by the Natural Sciences and Engineering Research Council of Canada.

[1] J. Ford and G. Mantica, Am. J. Phys. 60, 1086 (1992).

[2] The term relaxation denotes the evolution of all initial distribution to the same stationary final distribution. In the case of the cat map, this is the constant distribution in phase space.
[3] W. Zurek, Phys. Today 46, 81 (1993); A. R. Kolovsky, Phys. Rev. Lett. 76, 340 (1996).

[4] J. Wilkie and P. Brumer, Phys. Rev. E 49, 1968 (1994).

[5] V.I. Arnold and A. Avez, Ergodic Problems of Classical Mechanics (Addison-Wesley, New York, 1989).

[6] F. Izrailev, Phys. Rep. 196, 299 (1990).

[7] C. Jaffé and P. Brumer, J. Phys. Chem. 88, 4829 (1984); J. Chem. Phys. 82, 2330 (1985); C. Jaffé, S. Kanfer, and P. Brumer, Phys. Rev. Lett. 54, 8 (1985).

[8] See S.R. De Groot and L.G. Suttorp, Foundations of Electrodynamics (North-Holland, Amsterdam, 1972) for an excellent review of the Wigner-Weyl technique.

[9] J. Ford, G. Mantica, and G. H. Ristow, Physica (Amsterdam) 50D, 493 (1991).

[10] J. H. Hannay and M. V. Berry, Physica (Amsterdam) 1D, 267 (1980); J.P. Keating, Ph.D. thesis, University of Bristol, 1989 (unpublished); M. Degli Esposti, S. Graffi, and S. Isola, Commun. Math. Phys. 167, 471 (1995).

[11] J. Wilkie, Ph.D. dissertation, University of Toronto, 1994 (unpublished).

[12] Wave packets were initialized in phase space, Fourier transformed, and then propagated via Eq. (5) or Eq. (4) using a $512 \times 512$ grid; various accuracy checks were performed using a $1024 \times 1024$ sized grid. The classical Fourier trajectories are hyperbolic; thus the Fourier components leave any finite grid rapidly. This is the major source of computational error classically and in the quantal $\alpha \rightarrow 0$ regime. Dynamics in the deep quantal regime $(\alpha \rightarrow 1)$ do not exhibit any Fourier space hyperbolicity, thus avoiding the associated accuracy problems.

[13] J. S. Hutchinson and R. E. Wyatt, Phys. Rev. A 23, 1567 (1981).

[14] One Gaussian has parameters $\lambda=1, x_{0}=0.00712, p_{0}=$ $0.00904, \sigma_{x}=0.4153, \sigma_{p}=0.3841$; the second Gaussian is identical except for $x_{0}=0.00702$. The values of $\alpha$ for this set of computations were chosen arbitrarily in the near-classical regime. The parameters for the Gaussians were also chosen arbitrarily, constrained solely to be initially well localized while satisfying the uncertainty principle.

[15] The distance between two centroids, or between two point trajectories, is bounded due to the finite phase-space volume. Reaching this limit is what we term saturation. 